\title{
Weak Ferromagnetic Property and Electromagnetic Waves Absorption Characteristic of $\mathrm{La}_{(1-x)} \mathrm{Ba}_{x} \mathrm{MnO}_{3}$
}

\author{
E. Sukirman, Y. Sarwanto, W. Ari Adi, A. Insani, and Y. Fachmi Buys
}

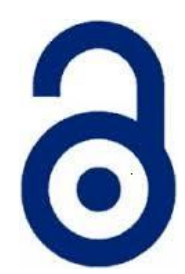

Received: 23 May 2019

Accepted: 30 August 2019

Published: 27 September 2019

Publisher: Deer Hill Publications

(C) 2019 The Author(s)

Creative Commons: CC BY 4.0

\begin{abstract}
The weak ferromagnetic property and the electromagnetic waves absorption characteristic of $\mathrm{La}_{(1-x)} \mathrm{Ba}_{\times} \mathrm{MnO}_{3}(\mathrm{LBMO})$ compounds have been investigated. The samples of $\mathrm{LBMO}$ that are $\mathrm{LaMnO}_{3}(\mathrm{SO}), \mathrm{La}_{0.9} \mathrm{Ba}_{0.1} \mathrm{MnO}_{3}(\mathrm{~S} 1) ; \mathrm{La}_{0.8} \mathrm{Ba}_{0.2} \mathrm{MnO}_{3}$ (S2); and $\mathrm{La}_{0.7} \mathrm{Ba}_{3} \mathrm{MnO}_{3}$ (S3) were synthesized using high energy milling (HEM) method. Samples were characterized by means of XRD (X-ray diffractometer), HRPD (high-resolution powder neutron diffractometer), EDS (energy dispersive $X$-ray spectroscopy, VSM (vibrating sample magnetometer), and VNA (vector network analyzer). There is no magnetic ordering of ferromagnetic in S1 and S2 samples due to the Ba occupation factors of both less than 0.2 . The Ba content in the $S 3$ sample is greater than 0.2 , hence the ferromagnetic property of the compound is not so visible with the VSM as well as the VNA. The absorption characteristics of electromagnetic waves using VNA indicated that there is an absorption of EM waves in the frequency range between $8-12 \mathrm{GHz}$ with almost the same peak frequency for all four samples at $10.8 \mathrm{GHz}$ with the absorption of around $5 \mathrm{~dB}$. The existence of a weak ferromagnetic property can be detected clearly using HRPD. Neutron diffraction as a probe can observe the magnetic structure accurately even in a material having a weak ferromagnetic property.
\end{abstract}

Keywords. La-manganite, Ba-substitution, cystal structure, electromagnetic wave absorber, weak ferromagnetic.

\section{INTRODUCTION}

Generally, the parent compound, $\mathrm{LaMnO}_{3}$ with perovskite $\mathrm{ABO}_{3}$ structure has antiferromagnetic insulator properties in which $\mathrm{Mn}$ is present in a single oxidation state $\left(\mathrm{Mn}^{+3}\right)$. It is found that the stable phase is the antiferromagnetic Atype, which corresponds to the ferromagnetic order of the manganese ions in the basal planes (a, b) and antiferromagnetic order of these ions between these planes along the $c$ axis [1]. The conductivity of rare-earth manganese oxides is enhanced to approach that of metal or semiconductor from the insulating state when doped and their colossal magnetoresistance effect (CMR) is remarkable [2]. Therefore, they are fascinating to be studied and applied on a large scale due to their unusual electromagnetic properties [3-5]. Besides that due to the unusual magnetic and electronic properties of the $\mathrm{LaMnO}_{3}$ compound, one can develop a very good electromagnetic wave absorbing device [6-8].

Luckily, the desired properties of $\mathrm{LaMnO}_{3}$ can be tuned easily by partial substitution of the trivalent rare-earth element $\left(\mathrm{La}^{3+}\right)$ with divalent alkaline earth elements, such as $\mathrm{Ba}^{2+}$ forming a new compound of $\mathrm{La}_{(1-\times)} \mathrm{Ba}_{\times} \mathrm{MnO}_{3}$ (LBMO). The property as a microwave absorber of this manganite is usually obtained by varying the concentration of $\mathrm{Ba}^{2+}$ element [3]. Substitutions at the trivalent rare earth site (A-site) by a divalent alkaline earth metal ion like $\mathrm{Ca}, \mathrm{Sr}, \mathrm{Ba}$ or $\mathrm{Pb}$ causes part of the $\mathrm{Mn}^{+3}$ are oxidized to $\mathrm{Mn}^{+4}$ ions and transforms this compound to a ferromagnetic metal [9]. In order, the electric charge of the compound to remain neutral, a number of $\mathrm{Mn}^{3+}$ ions donate some of its electrons to $\mathrm{Mn}^{4+}$ and therefore formed a mixed valence system of $\left[\boldsymbol{L a}_{(\mathbf{1}-\boldsymbol{x})}^{3+} \boldsymbol{B a}_{\boldsymbol{x}}^{2+}\right]\left[\mathbf{M n}_{(\mathbf{1 - y})}^{3+} \boldsymbol{M} \boldsymbol{n}_{\boldsymbol{y}}^{4+}\right] \boldsymbol{O}_{\mathbf{3}}^{2-}$. The ferromagnetic behaviour occurred due to magnetic interactions between $\mathrm{Mn}^{3+}$ and $\mathrm{Mn}^{4+}$ ions through the doubleexchange mechanism [10].

\footnotetext{
E. Sukirman ${ }^{1} \bowtie$, Y. Sarwanto 1 , W. Ari Adi', A. Insani ${ }^{1}$ and Y. F. Buys ${ }^{2}$

${ }^{1}$ Centre for Science and Technology of Advance Materials-BATAN

Puspiptek, Serpong 15314, Indonesia.

E-mail: skm2792@batan.go.id
}

2University of Malaya, Jalan Universiti, 50603 Kuala Lumpur

Wilayah Persekutuan Kuala Lumpur, Malaysia.

E-mail: yose@um.edu.my

Reference: Sukirman, E., Sarwanto, Y., Ari Adi, W., Insani, A., and Buys, F. Y. (2019). Weak Ferromagnetic Property and Electromagnetic Waves Absorption Characteristic of $\mathrm{La}_{(1-x)} \mathrm{Ba}_{x} \mathrm{MnO}_{3}$. International Journal of Engineering Materials and Manufacture, 4(3), 96-106 
The previous studies [11] showed that a strong ferromagnetism of LBMO compound occurred at $x \approx 0.2$, and at $x>$ 0.2 the properties of ferromagnetic materials become weak, while at $x<0.2$ the material becomes antiferromagnetic at room temperature. The purpose of this research is to determine the crystal structure, and to observe a weak ferromagnetic property of magnetic material in relation with the electromagnetic waves absorption characteristic of $\mathrm{La}(1-x) \mathrm{Ba}_{\times} \mathrm{MnO}_{3}$ (LBMO) compounds. It will be carried out using the neutron diffraction method, under the assumption that it could not be done by any other method. No studies have ever been done so far to prove the problem. In this study, the LBMO samples were synthesized by solid-state reaction method using High Energy Milling (HEM) process.

\section{METHODOLOCY}

The samples were prepared by using solid- state reaction method to the raw materials of $\mathrm{La}_{2} \mathrm{O}_{3}, \mathrm{BaCO}_{3}$, and $\mathrm{MnO}_{2}$, where the purity of $\mathrm{La}_{2} \mathrm{O}_{3}$ raw material is $99.5 \%$ from local products and the remaining two raw materials have more than $99.9 \%$ purity from Merck products. The solid reaction in this research was conducted through high energy milling (HEM) process with the same procedure as before [12]. Subsequently, the precursors were sintered at $1200^{\circ} \mathrm{C}$ for 5 hours at a heating rate of around $49^{\circ} \mathrm{C} / \mathrm{min}$ and the cooling was carried out naturally to room temperature in the furnace.

It was synthesized four kinds of samples of $\mathrm{La}_{1-x} \mathrm{Ba}_{\times} \mathrm{MnO}_{3}$ that are $\mathrm{LaMnO}_{3}$ (SO), $\mathrm{La}_{0.9} \mathrm{Ba}_{0.1} \mathrm{MnO}_{3}$ (S1), Lao.8Ba0.2 $\mathrm{MnO}_{3}$ (S2), and $\mathrm{La}_{0.7} \mathrm{Ba}_{0.3} \mathrm{MnO}_{3}$ (S3). Phase characterization was then performed with X-ray diffractometer (XRD), type of PW1710 Philips with cooper anode tube, $\lambda=1.5406 \AA$, the data were collected in the scattering angular range of $10^{\circ}$ up to $100^{\circ}$ in the interval of $0.02^{\circ}$ at room temperature. The phase was also characterized using high-resolution powder neutron diffractometer (HRPD) with $\lambda=1.8216 \AA$. The sample was loaded into a cylindrical vanadium holder. The data was collected in the scattering angular range of $2.5^{\circ}$ up to $157^{\circ}$ in the interval of $0.05^{\circ}$ at room temperature. The XRD data were analysed with the help of general structure analysis system (CSAS) and the neutron diffraction data was refined using the FullProof software. The elementary analysis was performed using scanning electron microscope (SEM) and energy dispersive X-ray spectroscopy (EDS) of JEOL instrument.

The electromagnetic waves absorption characteristics of the samples were analysed by using vector network analyser (VNA) of ADVANTEST R3770 types with the frequency range of $300 \mathrm{kHz}-20 \mathrm{GHz}$. The magnetic properties of materials were characterized using vibrating sample magnetometer (VSM), Oxford Type 1.2 Hin method. Measurement of magnetic properties of materials was made in an applied magnetic field to a maximum value of 1 Tesla. VSM measurements were carried out at room temperature. Measurements, XRD, HRPD, VSM and SEM / EDS were performed at the Centre for Advanced Materials Science and Technology (PSTBM), BATAN, Puspiptek, Setu, Tangerang Selatan. While the measurements of VNA were done at the Centre for Electronic Research and Telecommunications (PPET), LIPI, Bandung.

\section{RESULTS AND DISCUSSIONS}

The Rietveld analysis based on the X-ray diffraction data using GSAS software from the $\mathrm{LaMnO}_{3}, \mathrm{La}_{0.9} \mathrm{Ba}_{0.1} \mathrm{MnO}_{3}$, $\mathrm{La}_{0.8} \mathrm{Ba}_{0.2} \mathrm{MnO}_{3}$, and $\mathrm{La}_{0.7} \mathrm{Ba}_{0.3} \mathrm{MnO}_{3}$. Samples were showed in Figure 1(SO), 1(S1), 1(S2) and 1(S3), respectively. The diffraction pattern of $\mathrm{SO}$ exhibited a single phase diffraction profile within the limits of accuracy of the tool. The dots are the observed intensity, the solid line was the calculated intensity, and the difference pattern was shown at the bottom of the chart. The SO sample crystallizes into the space group: 112/a1, with $\chi^{2}=1.3$ and the lattice parameters: $a=7.7839(5) \AA, b=5.5288(4) \AA, c=5.4781(3) \AA, \alpha=90^{\circ}, \beta=90.746(2)^{\circ}, \gamma=90.0^{\circ}$ and volume, $V=235.73(4)$ $\AA^{3}$. The coordinates of the atomic fractions were shown in Table 1. The smoothing on the occupancy factor of oxygen atoms caused their value to be greater than 1.0. According to previous research [11], the SO sample should display the properties as a paramagnetic material. This will be proved by measurement using VSM.

The diffraction pattern of $S 1$ sample which was the result of analysis by Rietveld method was shown in Figure $1(S 1)$. The $S 1$ samples also showed a single phase diffraction pattern within the limits of accuracy of the tool. It was obtained a better fitting result with $\chi^{2}=1.3$. The $S 1$ sample crystallized also into the space group: 112/a1 in accordance with the results of previous research [11], the lattice parameters: $a=7.8142(9) \AA, b=5.5462(6) \AA, c=5.4993(6)$ $\AA, \alpha=90^{\circ}, \quad \beta=90.593(5)^{\circ}, \gamma=90^{\circ}$ and volume, $V=238.33(7) \AA^{3}$. The atomic fraction coordinates $\left(x_{j}, y_{j}, z_{j}\right)$, and the atomic occupancy factor $\left(g_{j}\right)$ of $S 1$ sample was indicated in Table 2 . The mole fraction of La and Ba that were 0.86, and 0.105, respectively, correspond to the weighed mole fraction on the synthesis that was 0.9 and 0.1 , respectively. The occupation factors of $\mathrm{Mn}, \mathrm{O}(1)$ and $\mathrm{O}(2)$ were not refined, because the smoothing caused their value to be greater than 1.0 .

Table 1. The coordinates of atomic fractions $\left(x_{j}, y_{j}, z_{j}\right)$, and atomic occupancy factor $\left(g_{j}\right)$ of SO sample.

\begin{tabular}{ccccc}
\hline Atom & $g_{j}$ & $x_{j}$ & $y_{j}$ & $z_{j}$ \\
\hline La & $0.810(1)$ & 0.25 & $0.5066(6)$ & 0.0 \\
Mn & $0.85(2)$ & 0.0 & 0.0 & 0.0 \\
O(1) & 1.0 & 0.25 & 0.0 & 0.0 \\
O(2) & 1.0 & $-0.013(2)$ & $0.198(3)$ & $0.292(4)$
\end{tabular}




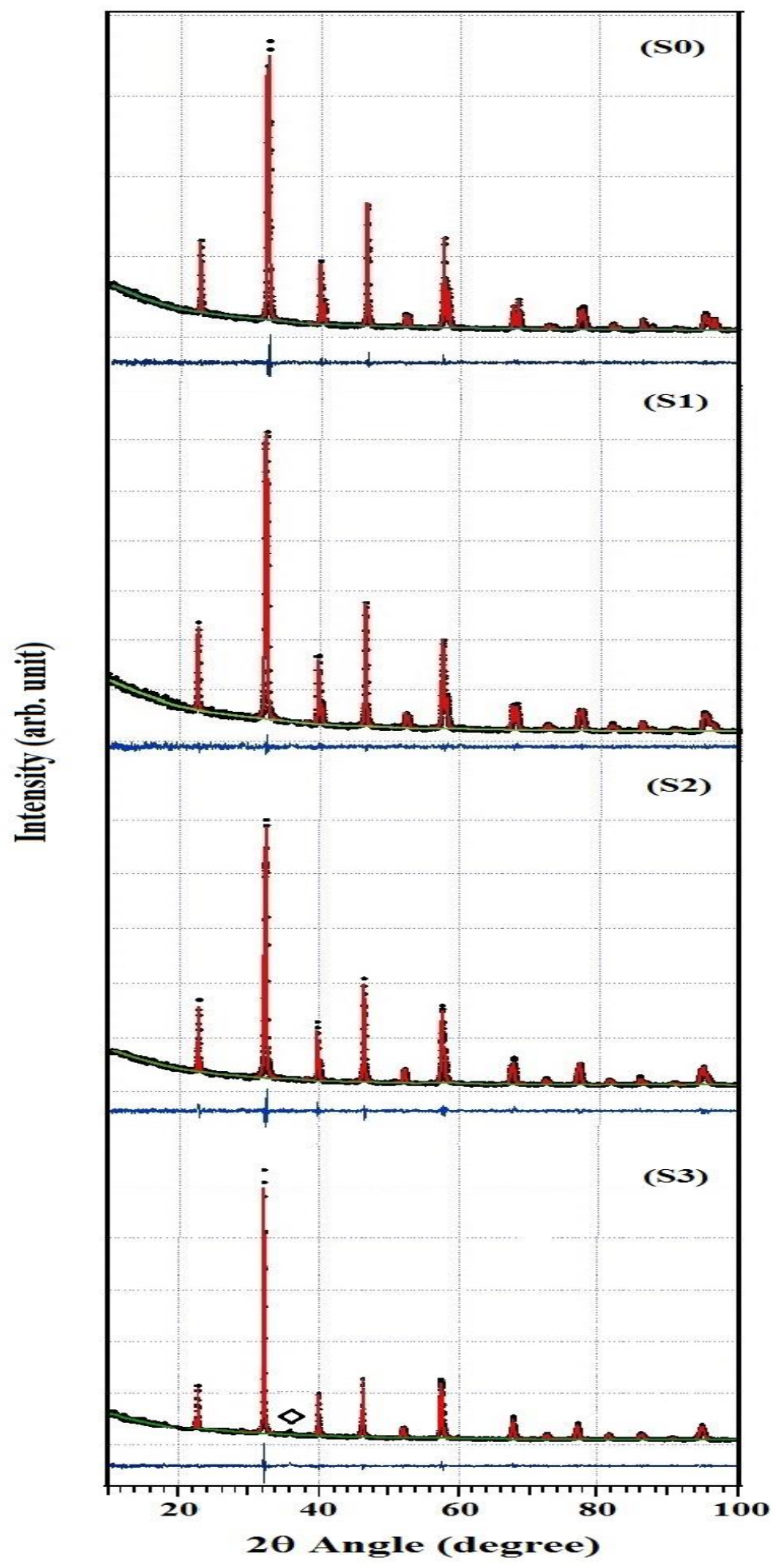

Figure 1. The X-ray diffraction pattern of $\mathrm{LaMnO}_{3}$ (SO), $\mathrm{La} \mathrm{ga}_{0.9} \mathrm{Ba}_{0.1} \mathrm{MnO}_{3}(\mathrm{~S} 1)$, $\mathrm{La} 0.8 \mathrm{Ba}_{0.2} \mathrm{MnO}_{3}\left(\mathrm{~S} 2\right.$ ), and $\mathrm{La}_{0.7} \mathrm{Ba}_{0.3} \mathrm{MnO}_{3}$ (S3).

Based on the results of previous research [11], the S1 sample should display the properties as an antiferromagnetic or a paramagnetic instead of ferromagnetic material due to the occupation factor of Ba was smaller than 0.2. This will be proved by measurement using VSM. The pattern of diffraction after analysis by Rietveld method of $\$ 2$ sample was showed in Figure 1(\$2). It appeared on the figure that the sample displayed a single phase diffraction pattern to the 
extent of the accuracy of the tool. The $\$ 2$ sample crystallized into the space group: $112 / c 1$ in accordance with the results of previous research [11], with the goodness of fitting, $\chi^{2}=1.2$, the lattice parameters: $a=5.5118(3) \AA, b=$ 5.5401(4) $\AA, c=7.8244(4) \AA, \alpha=90^{\circ}, \beta=90.447(6)^{\circ}, \gamma=90^{\circ}$ and volume, $V=238.92(1) \AA^{3}$. The atomic fraction coordinates $\left(x_{j}, y_{j}, z_{j}\right)$, and the atomic occupancy factor $\left(g_{j}\right)$ of $\$ 2$ sample were indicated in Table 3 . The occupation factor of oxygens was not refined, because the smoothing caused their value to be greater than 1.0.

The atomic fraction coordinates of $\mathrm{O}(2)$ were not refined, because when those parameters were smoothed caused the chi- square enlarged. It appeared in Table 3 that the S2 lacked La and Ba atoms in its unit cell that were 0.65 and 0.17 , respectively. The mole fraction of $\mathrm{La}$ and $\mathrm{Ba}$ atoms did not correspond to the weighed mole fraction on the synthesis that was 0.8 and 0.2 , respectively. The previous studies [11] showed that a strong ferromagnetism of LBMO compound occurred when the $\mathrm{Ba}$ occupation factor of $x \approx 0.2$, and at $x<0.2$ the material becomes antiferromagnetic. Thus, S2 sample should also display the properties as an antiferromagnetic or a paramagnetic material instead of ferromagnetic one due to the occupation factor of $\mathrm{Ba}^{2+}$ ion was smaller than 0.2. This will be proved by measurement using VSM.

The diffraction pattern after being analysis by the Rietveld method of $\$ 3$ sample was shown in Figure $1(\$ 3)$. It appeared on the figure that there was a peak of foreign phase at $2 \theta \approx 36^{\circ}$, where this peak was marked with symbol $\checkmark$. Based on XRD data, the presence of this foreign phase cannot be analysed, possibly because the amount is smaller than the XRD detection limit. Table 4 was the coordinates of atomic fractions $\left(x_{j}, y_{j}, z_{j}\right)$, and atomic occupantion factor $\left(g_{j}\right)$ of $\$ 3$ sample. It appeared in this table that the occupation factor $\left(g_{j}\right)$ of La and Ba atoms in its unit cell ware 0.61 and 0.22 , respectively. Thus, the $\$ 3$ sample should displays a weak ferromagnetic property due to $g_{j}$ of $\mathrm{Ba}^{2+}$ ion was greater than 0.2 , this will be proved later.

The $\$ 3$ sample crystallized into the space group: R-3c in accordance with the results of previous research [13], with the goodness of fitting, $\chi^{2}=1.6$ and the lattice parameters: $a=b=5.5301$ (7) $\AA, c=13.532(3) \AA, \alpha=\beta=$ $90^{\circ}, \gamma=120^{\circ}$, volume, $V=358.41(9) \AA^{3}$. The occupation factor of oxygen was not refined, because the smoothing caused its value to be greater than 1.0.

Table 2. The coordinates of atomic fractions $\left(x_{j}, y_{j}, z_{j}\right)$, and atomic occupancy factor $\left(g_{j}\right)$ of $S 1$ sample.

\begin{tabular}{ccccc}
\hline Atom & $g_{j}$ & $x_{j}$ & $y_{j}$ & $z_{j}$ \\
\hline La & $0.86(1)$ & 0.25 & $0.502(1)$ & 0.0 \\
Ba & $0.105(4)$ & 0.25 & $0.502(1)$ & 0.0 \\
Mn & 1.0 & 0.0 & 0.0 & 0.0 \\
O(1) & 1.0 & 0.25 & 0.0 & 0.0 \\
O(2) & 1.0 & $-0.017(4)$ & $0.204(4)$ & $0.281(5)$ \\
\hline
\end{tabular}

Table 3. The coordinates of atomic fractions $\left(x_{j}, y_{j}, z_{j}\right)$, and atomic occupancy factor $\left(g_{j}\right)$ of $\$ 2$ sample.

\begin{tabular}{ccccc}
\hline Atom & $g_{j}$ & $x_{j}$ & $y_{j}$ & $z_{j}$ \\
\hline La & $0.65(1)$ & 0.0 & $0.499(2)$ & 0.75 \\
Ba & $0.17(3)$ & 0.0 & $0.499(2)$ & 0.75 \\
Mn & $0.853(9)$ & 0.0 & 0.0 & 0.0 \\
O(1) & 1.0 & 0.0 & $0.056(3)$ & 0.25 \\
O(2) & 1.0 & 0.251 & 0.249 & -0.03 \\
\hline
\end{tabular}

Table 4. The coordinates of atomic fractions $\left(x_{j}, y_{j}, z_{j}\right)$, and atomic occupancy factor $\left(g_{j}\right)$ of $\$ 3$ sample.

\begin{tabular}{ccccc}
\hline Atom & $g_{j}$ & $x_{j}$ & $y_{j}$ & $z_{j}$ \\
\hline La & $0.61(1)$ & 0.0 & 0.0 & 0.25 \\
Ba & $0.22(1)$ & 0.0 & 0.0 & 0.25 \\
Mn & $0.92(2)$ & 0.0 & 0.0 & 0.0 \\
O & 1.0 & $0.480(6)$ & 0.0 & 0.25 \\
\hline
\end{tabular}

Figure 2 showed the EDS spectrum of SO, S1, S2, and S3. The four samples showed an identical EDS spectrum. Table 5 was the mass fraction of the elements in $S 0, S 1, S 2$, and $\$ 3$ samples which were based on the number of counts each peak of the EDS spectrum. Then the mass fraction of Ba elements in $\$ 1, S 2$ and $\$ 3$ were then converted into the mole fraction of Ba elements, the result was showed in Table 6.

It appeared in Table 6 that the mole fraction of Ba elements in S1 and S2 showed by EDS data in accordance to data of XRD and also in accordance to the one on weighing, that was 0.1 and 0.2 , respectively. This means that on 
both samples, the $\mathrm{Ba}$ atoms have entered into the unit cell of $\mathrm{LaMnO}_{3}$. The mole fraction of the XRD data is the mole fraction per unit cell, whereas the mole fraction of the EDS data is the one of the element present in the sample. The mole fraction of the Ba element in S3 showed by EDS data was in accordance with the one on weighing, i.e., 0.3, but was incompatible with XRD data, i.e., 0.2. It was concluded that there was about 0.1 mole of Ba elements not entering into the unit cell of $\mathrm{LaMnO}_{3}$, but forming a new phase. A foreign peak on Figure 1 (S3) appearing at $2 \theta \approx$ $36^{\circ}$ was assumed to belong to the new phase formed by Ba elements.

Table 5. The mass fraction of O, Mn, Ba and La elements in SO, S1, S2, and S3 samples.

\begin{tabular}{|c|c|c|c|c|c|}
\hline \multirow[b]{2}{*}{ Element } & \multirow[b]{2}{*}{$(\mathrm{keV})$} & \multicolumn{2}{|c|}{ Energy } & \multicolumn{2}{|c|}{ Mass fraction (\%) } \\
\hline & & SO & S1 & S2 & S3 \\
\hline \multirow[t]{2}{*}{$\mathrm{O}$} & 0.525 & 18.68 & 17.88 & 22.23 & 18.94 \\
\hline & & \pm 0.09 & \pm 0.07 & \pm 0.06 & \pm 0.07 \\
\hline \multirow[t]{2}{*}{ Mn } & 5.894 & 21.72 & 23.65 & 19.76 & 20.94 \\
\hline & & \pm 0.25 & \pm 0.19 & \pm 0.18 & \pm 0.21 \\
\hline \multirow[t]{2}{*}{$\mathrm{Ba}$} & 4.464 & - & 5.67 & 10.06 & 17.16 \\
\hline & & & \pm 0.25 & \pm 0.23 & \pm 0.27 \\
\hline \multirow[t]{2}{*}{ La } & 4.648 & 59.60 & 52.80 & 47.95 & 42.96 \\
\hline & & \pm 0.35 & \pm 0.29 & \pm 0.27 & \pm 0.31 \\
\hline
\end{tabular}

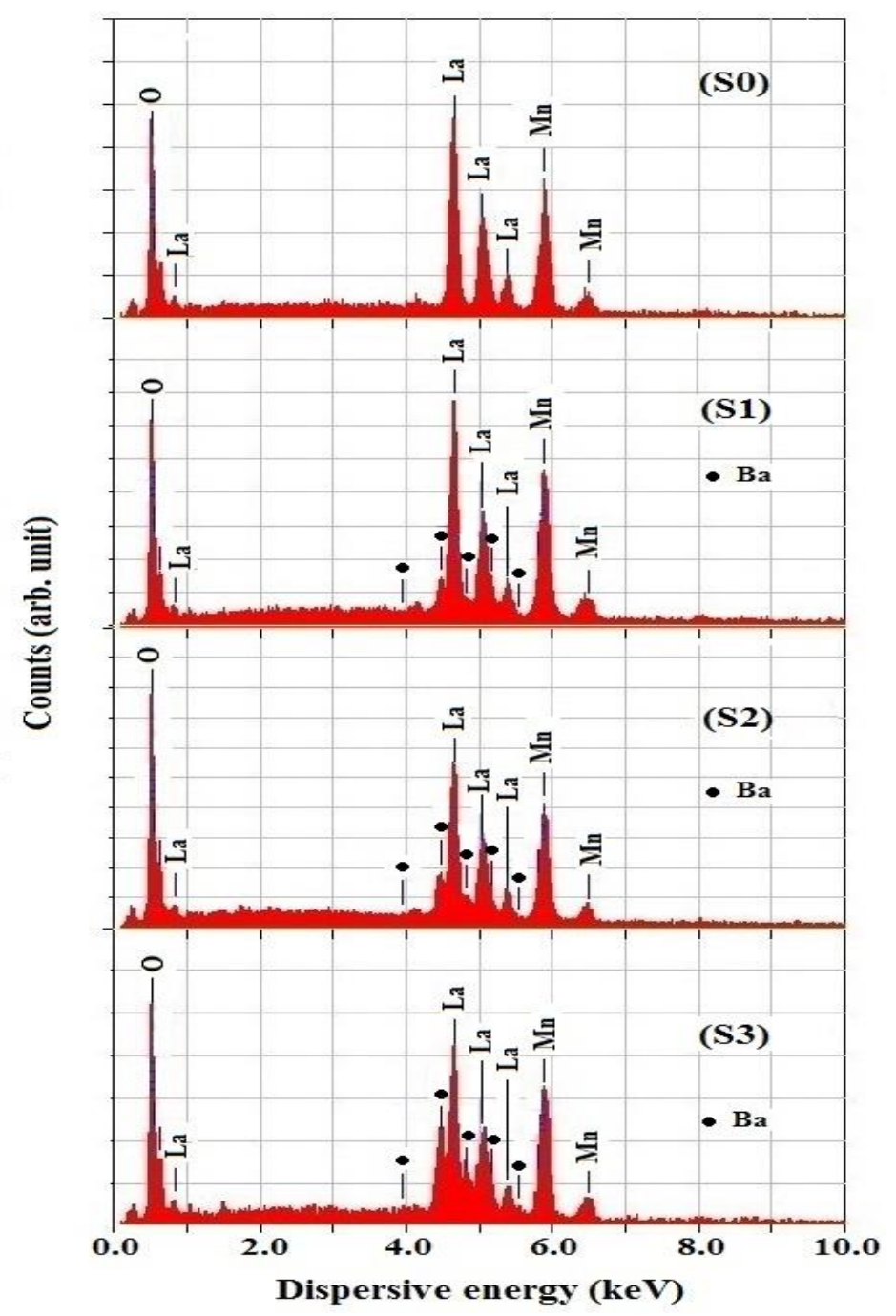

Figure 2. The EDS spectrum of $\mathrm{LaMnO}_{3}$ (SO), $\mathrm{La}_{0.9} \mathrm{Ba}_{0.1} \mathrm{MnO}_{3}$ (S1), $\mathrm{La}_{0.8} \mathrm{Ba}_{0.2} \mathrm{MnO}_{3}$ (S2), and $\mathrm{La}_{0.7} \mathrm{Ba}_{0.3} \mathrm{MnO}_{3}$ (S3). 
Table 6. The mole fraction of Ba element in $\$ 1, \$ 2$, and $\$ 3$ samples result from the conversion of its mass fraction.

\begin{tabular}{lcccc}
\hline & & \multicolumn{2}{c}{ Mole fraction of Ba element. } \\
\hline Sample & & weighing & XRD & EDS \\
& S1 & 0.1 & $0.105(4)$ & $0.098(2)$ \\
& S2 & 0.2 & $0.17(3)$ & $0.170(2)$ \\
& S3 & 0.3 & $0.22(1)$ & $0.283(2)$ \\
\hline
\end{tabular}

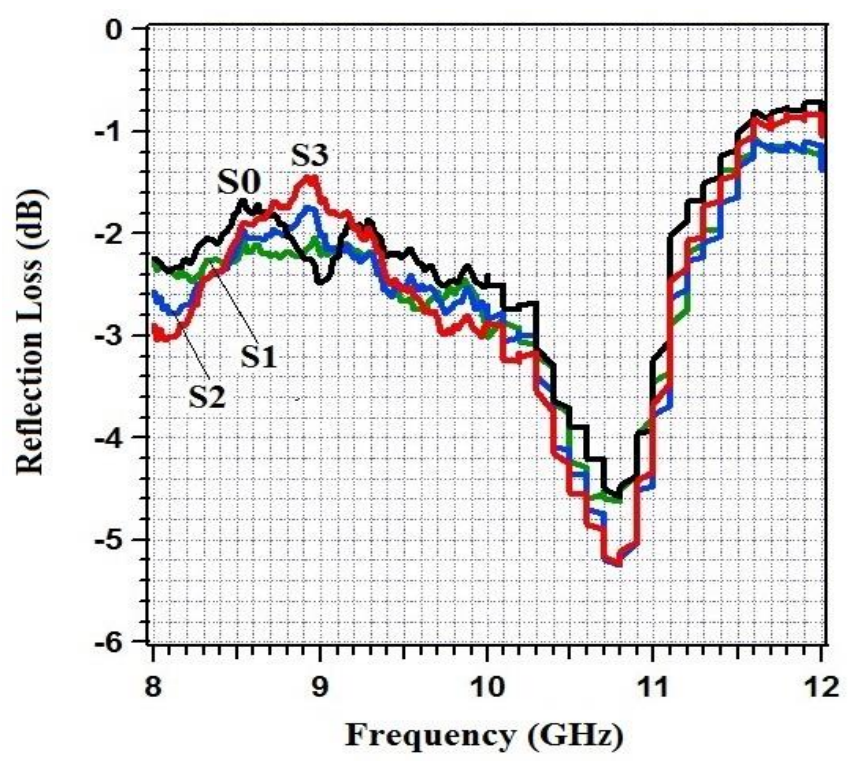

Figure 3. The microwave absorbing characteristics of the $\mathrm{SO}, \mathrm{S1}, \mathrm{S2}$ and $\mathrm{S3}$ samples in the range of $2-12 \mathrm{GHz}$.

The interaction of the ceramics with electromagnetic waves characterized by using an HP8720B vector network analyser in the frequency range of $2-12 \mathrm{GHz}$ was shown in Figure 3 . The results revealed that the electromagnetic wave absorption characteristics of $\$ 0, S 1, \$ 2$, and $\$ 3$ were almost the same, i.e., showing the same reflection loss peaks of around $-5 \mathrm{~dB}$ at a frequency of $10.8 \mathrm{GHz}$. The microwave absorption primarily resulted from magnetic losses caused by magnetization relaxation, domain wall resonance, and natural resonance [14]. This means that all four samples have the same magnetic properties, i.e., as an antiferromagnetic or a paramagnetic materials, because they do not display significant reflection loss, where the previous research [11] showed only manganite with the magnetic ordering of ferromagnetic has the best ferromagnetic properties and therefore display the best characteristic in absorbing an electromagnetic wave.

The curves showing the relation between magnetization, $M$ to magnetic field, $\mathrm{H}$, commonly called magnetization curves, for the four samples were shown in Figure 4. It was exhibited that SO, S1, and S2 samples displayed a typical pattern for the antiferromagnetic materials, i.e., displaying the linear curve of $M$ vs $H$. While the $M$ vs. $H$ curve of S3 does not perfectly display a straight line, but slightly curved to form an incomplete $S$ letter. This is in accordance with the results of previous studies [11] which showed that the LBMO compounds, whose the Ba occupation factors of $\mathbf{g}_{j}$ less than, or greater than 0.2 were antiferromagnetic, or weak ferromagnetic materials, respectively.

The presence of a paramagnetic, antiferromagnetic or ferromagnetic phase in LBMO samples was then investigated by neutron diffraction technique using the Rietveld analytical methods with the help of FullFrof software. Based on the XRD data, the three samples of SO, S1, and $\$ 2$ have the monoclinic crystal structure, while $\$ 3$ sample crystallized with hexagonal structure. The analysis of neutron diffraction data, therefore, was performed on SO only because the three samples are identical. Analysis of neutron diffraction data was also carried out on $\$ 3$ with a purpose besides to confirm the presence of antiferromagnetic or weak ferromagnetic phases also to confirm the presence of foreign phase.

First, it was carried out the analysis of nuclear structure with input parameters equal to the input parameters in XRD data analysis using CSAS software. The results show that all of the diffraction peaks coincide with the calculation results based on the nuclear peaks (Figure 5). This means there are no additional peaks due to scattering from the magnetic phase. So the magnetic structure would have a propagation vector $k=(0,0,0)$. As the propagation vector is $k=(0,0,0)$, the magnetic unit cell is identical to the nuclear cell. 
Next, the BasIRreps program was employed to determine the basis vectors of the irreducible representations (IRreps) of the propagation vector group $\left(G_{k}\right)$. With the help of this program one can determine the appropriate magnetic symmetry and the magnetic rotation vectors. The output of the calculation for the basic functions of IRreps corresponding to the experimental magnetic structure of $\mathrm{LaMnO}_{3}$ with space group: 112/a1 are a basic function of IRreps (1) for antiferromagnetic structures (Table 7) and IRreps (3) for ferromagnetic structures (Table 8).

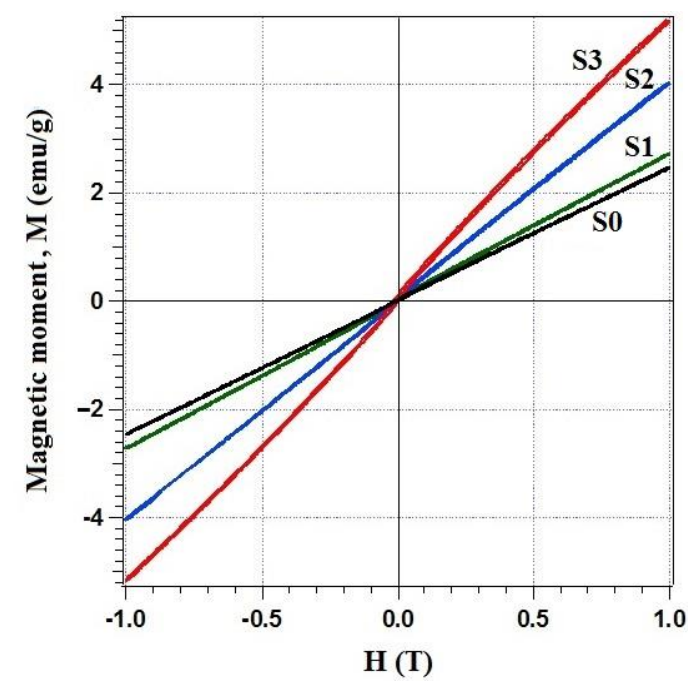

Figure 4. The curve for magnetization, $\mathrm{M}$ to magnetic field, $\mathrm{H}$ from sample $\mathrm{LaMnO}_{3}$ (SO), $\mathrm{La}_{0.9 \mathrm{Ba}} \mathrm{Ba}_{1} \mathrm{MnO}_{3}(\mathrm{~S} 1)$, $\mathrm{La}_{0.8} \mathrm{Ba}_{0.2} \mathrm{MnO}_{3}(\mathrm{~S} 2)$, and $\mathrm{La}_{0.7} \mathrm{Ba}_{0.3} \mathrm{MnO}_{3}$ (S3).

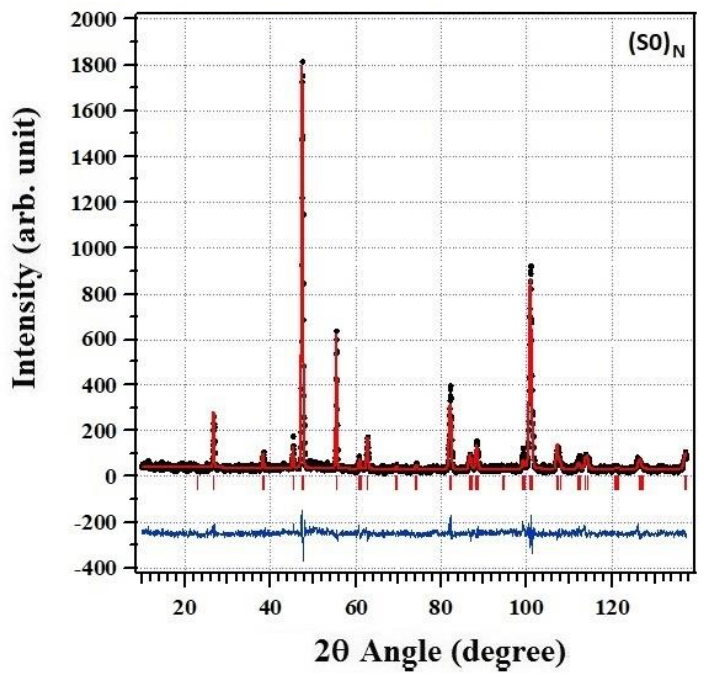

Figure 5. Neutron diffraction patterns of $\mathrm{LaMnO}_{3}(\mathrm{SO})$, the Rietveld analysis results based on the nuclear phase only without involving magnetic phase, with $\chi^{2}=1.9$

Table 7. Basis functions of Representation IRrep (1) of $\mathrm{LaMnO}_{3}$, space group: 112/a1, magnetic symmetry (SYMM), magnetic rotation vector $\left(S_{k}\right)$, fractional coordinates $\left(x_{j}, y_{j}, z_{j}\right)$ of $M n(1)$, and $M n(2)$ atoms.

\begin{tabular}{ccc}
\hline Atoms & $\operatorname{Mn}(1)$ & $\operatorname{Mn}(2)$ \\
\hline$\left(x_{j}, y_{j}, z_{j}\right)$ & $(0.0,0.0,0.0)$ & $(0.5,0.0,0.0)$ \\
SYMM & $(x, y, z)$ & $(-x+1 / 2, y,-z)$ \\
$S_{k}$ & $(u, v, w)$ & $(-u, v,-w)$ \\
\hline
\end{tabular}


Thus, analysis of the magnetic structure on $\mathrm{LaMnO}_{3}$ was carried out by trial and error, first input the data in Table 7 followed by the data in Table 8 to determine which data will give the smallest $\chi^{2}$. The chi-square of the neutron diffraction patterns of SO using the IRreps(1) and IRreps(3) are 1.8 and 2.6, respectively. Thus, SO has a magnetic ordering of antiferromagnetic, where the lattice of magnetic ions in the crystal breaks up into two sublattices along the a-axis having magnetic moments, $\mu=1.7(5) \mu_{B}$ in opposite directions (Figure 6). This result is in agreement with VSM data and in accord with the previous research indicated by Priyo Sardjono et. al. [11].

Figure 7 is the neutron diffraction patterns of 50 based on two phases, namely the nuclear-, and magnetic phase using basis functions of IRreps(1). The SO sample crystallizes into single phase of $\mathrm{LaMnO}_{3}$, space group: 112/a1, and the lattice parameters: $a=7.8217(7) \AA, b=5.5458(5) \AA, c=5.5230(6) \AA, \alpha=\gamma=90^{\circ}$, and $\beta=90.217(7)^{\circ}$. The vertical short lines closest to the horizontal axis indicate the position of the peaks of nuclear scattering, while the vertical short lines below them are the position of the peaks of the magnetic scattering. Table 9 was the coordinate of atomic fractions $\left(x_{j}, y_{j}, z_{j}\right)$, and atomic occupancy factors $\left(g_{j}\right)$ of $\mathrm{LaMnO}_{3}$ based on the neutron diffraction experiment.

Table 8. Basis functions of Representation IRrep (3) of $\mathrm{LaMnO}_{3}$, space group: 112/a1, magnetic symmetry (SYMM), magnetic rotation vector $\left(S_{k}\right)$, fractional coordinates $\left(x_{j}, y_{j}, z_{j}\right)$ of $M n(1)$, and $M n(2)$ atoms.

\begin{tabular}{lll}
\hline Atoms & $\operatorname{Mn}(1)$ & $\operatorname{Mn}(2)$ \\
\hline$\left(x_{j}, y_{j}, z_{j}\right)$ & $(0.0,0.0,0.0)$ & $(0.5,0.0,0.0)$ \\
SYMM & $(x, y, z)$ & $(-x+1 / 2, y,-z)$ \\
$S_{k}$ & $(u, v, w)$ & $(u,-v, w)$ \\
\hline
\end{tabular}

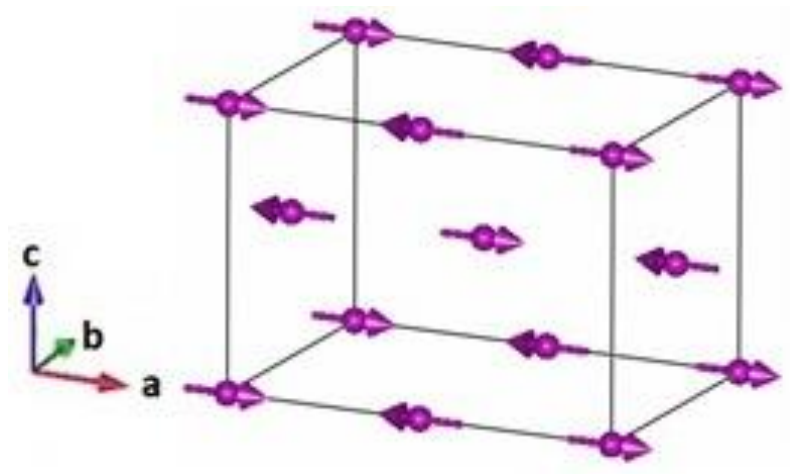

Figure 6. The antiferromagnetic structure of $\mathrm{LaMnO}_{3}$.

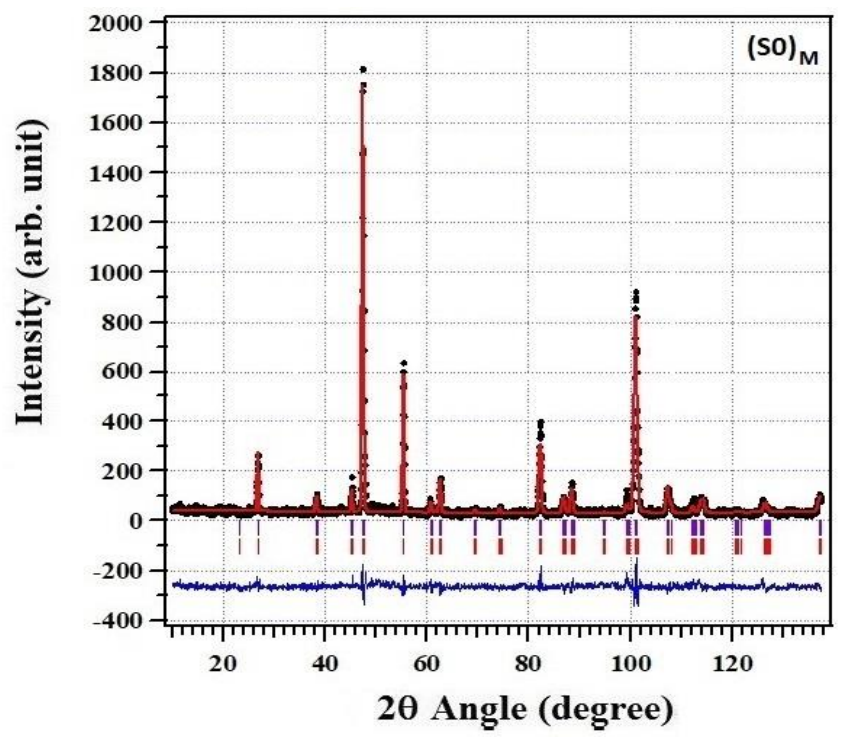

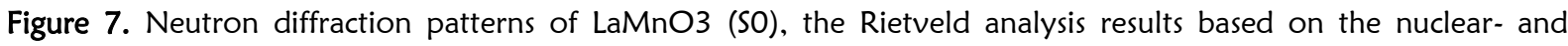
magnetic phase using basis functions of IRreps (1), with $\chi^{2}=1.8$. 
Table 9. The coordinates of atomic fractions $\left(x_{j}, y_{j}, z_{j}\right)$, and atomic occupancy factor $\left(g_{j}\right)$ of SO sample based on neutron diffraction data.

\begin{tabular}{ccccc}
\hline Atom & $g_{j}$ & $\mathrm{x}_{j}$ & $\mathrm{y}_{j}$ & $\mathrm{z}_{j}$ \\
\hline La & $0.4(7)$ & 0.25 & $0.509(2)$ & 0.0 \\
$\mathrm{Mn}$ & $0.6(7)$ & 0.0 & 0.0 & 0.0 \\
$\mathrm{O}(1)$ & $0.2(4)$ & 0.25 & 0.0 & 0.0 \\
$\mathrm{O}(2)$ & 1.0 & 0.0 & $0.220(1)$ & $0.267(2)$ \\
\hline
\end{tabular}

Table 10. Basis functions of Representation IRrep (1) of $\mathrm{LaMnO}_{3}$, space group: R-3c, magnetic symmetry (SYMM), magnetic rotation vector $\left(S_{k}\right)$, fractional coordinates $\left(x_{j}, y_{j}, z_{j}\right)$ of $M n(1)$, and $M n(2)$ atoms.

\begin{tabular}{ccc}
\hline Atoms & $M n(1)$ & $M n(2)$ \\
\hline$(x \mathbf{j}, \mathrm{yj}, \mathrm{zj})$ & $(0.0,0.0,0.0)$ & $(0.0,0.0,0.5)$ \\
SYMM & $(x, y, z)$ & $(\mathrm{y}, \mathrm{x},-\mathrm{z}+1 / 2)$ \\
Sk & $(0,0, \mathrm{u})$ & $(0,0,-\mathrm{u})$ \\
\hline
\end{tabular}

Table 11. Basis functions of Representation IRrep (3) of $\mathrm{LaMnO}_{3}$, space group: R-3c, magnetic symmetry (SYMM), magnetic rotation vector $\left(S_{k}\right)$, fractional coordinates $\left(x_{j}, y_{j}, z_{j}\right)$ of $M n(1)$, and $M n(2)$ atoms.

\begin{tabular}{ccc}
\hline Atoms & $\operatorname{Mn}(1)$ & $\operatorname{Mn}(2)$ \\
\hline$\left(\mathrm{x}_{\mathrm{j}}, \mathrm{y}_{\mathrm{j}}, \mathrm{z}_{\mathrm{j}}\right)$ & $(0.0,0.0,0.0)$ & $(0.0,0.0,0.5)$ \\
SYMM & $(\mathrm{x}, \mathrm{y}, \mathrm{z})$ & $(\mathrm{y}, \mathrm{x}, \mathrm{z}+1 / 2)$ \\
$S_{\mathrm{k}}$ & $(0,0, \mathrm{u})$ & $(0,0, \mathrm{u})$ \\
\hline
\end{tabular}

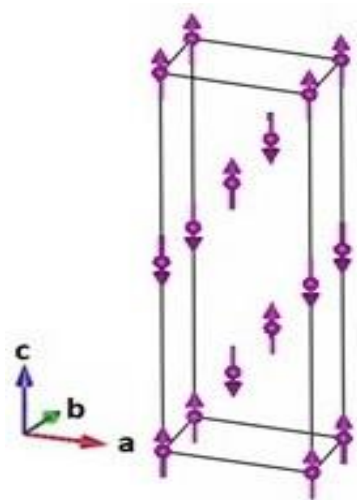

(a)

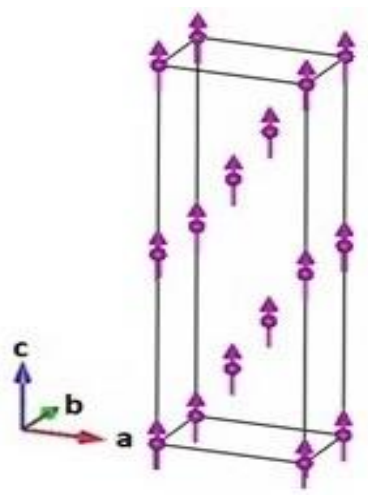

(b)

Figure 10. The antiferromagnetic structure (a), and ferromagnetic structure (b) of Ba-doped $\mathrm{LaMnO}_{3}(\mathrm{S3})$.

Furthermore, magnetic structural analysis of $\$ 3$ was done as for $\$ 0$ above. The output of the calculation for the basis functions of IRreps corresponding to the experimental magnetic structure of $\mathrm{LaMnO}_{3}$ with space group: R-3c are basis functions of IRreps(1) for antiferromagnetic structures (Table 10) and IRreps(3) for ferromagnetic structures (Table 11). The result of observation by means of Match software of $\$ 3$ shows the existence of a new phase of $\mathrm{BaLa}_{2} \mathrm{O}_{4}$. This X-ray diffraction data of the $\mathrm{BaLa}_{2} \mathrm{O}_{4}$ phase could not be analyzed by CSAS software, presumably due to its small content. The presence of $\mathrm{BaLa}_{2} \mathrm{O}_{4}$ phase was then observed by HRPD together with the nuclear and magnetic phases.

Analysis of the magnetic structure on S3 was carried out with the input parameters of Table 10 and Table 11 simultaneously. This step was carried out because in $\mathrm{S} 3$ other than the nuclear phase of $\mathrm{LBMO}$ and $\mathrm{BaLa}_{2} \mathrm{O}_{4}$ phase, it is also assumed that there are two magnetic phases, namely antiferromagnetic- and ferromagnetic phase. The analysis of neutron diffraction data using the Rieveld method utilizing FullProf software shows that $S 3$ has two magnetic phases, namely the ferromagnetic and antiferromagnetic phases with the chi-square of the neutron diffraction pattern of $S 3$ is 1.7. The sample of $S 3$ has magnetic ordering of antiferromagnetic and ferromagnetic with the magnetic moment, $\mu=1.9(2) \mu$ в (Figure 10).

The VSM data reveals that the $M$ vs. $\mathrm{H}$ curve of $\$ 3$ does not perfectly display a straight line, but slightly curved to form an incomplete $S$ letter. This means that $S 3$ is not a pure antiferromagnetic material. This material imperfection is apparently still detected by neutron diffraction methode as a material that has a composition of atoms such as 
ferromagnetic order, although weak. This result is in agreement with the results of previous research indicated by Priyo Sardjono et. al [11] and Sukirman et al [15].

Figure 11 is the neutron diffraction patterns of $\$ 3$ based on four phases, namely the nuclear-, antiferromagnetic-, ferromagnetic-, and $\mathrm{BaLa}_{2} \mathrm{O}_{4}$ phase after being analyzed with FullProf software with the goodness of fitting, $\chi^{2}=$ 1.7 and the coordinates of the atomic fractions was showed on Table 12. Rows of the vertical short lines closest to the horizontal axis indicate the position of the peaks of nuclear scattering, while the vertical short lines below them are the position of the peaks of the magnetic scattering, and the third row below is the peak positions of $\mathrm{BaLa}_{2} \mathrm{O}_{4}$ phase.

The atomic occupancy factors (gj) on Table 12 corresponded to the one on Table 4 which showed that the mole fraction of $\mathrm{La}$ and $\mathrm{Ba}$ atoms in the unit cell of the LBMO compound were each smaller than their value when weighed at the time of the synthesis. This was an evidence that some of the atoms of Ba and La did not enter into the unit cell of the compound, but form a new compound, i.e., $\mathrm{BaLa}_{2} \mathrm{O}_{4}$, Space Group: Pnam (No. 62), lattice parameters: $a=$ $10.621(2) \AA, b=12.466(3) \AA, c=3.7191(6) \AA, \alpha=\beta=\gamma=90^{\circ}$. Table 13 were the coordinates of atomic fraction $\left(\mathrm{x}_{\mathrm{j}}, \mathrm{y}_{\mathrm{j}}, \mathrm{z}_{\mathrm{j}}\right)$, and atomic occupancy factors $\left(\mathrm{g}_{\mathrm{j}}\right)$ of $\mathrm{BaLa}_{2} \mathrm{O}_{4}$ phase based on neutron diffraction data. It can be concluded that with the support of neutron diffraction data, the reflection loss versus frequency curve (Figure 3) and magnetization versus magnetic field curves (Figure 4) are both corresponding.

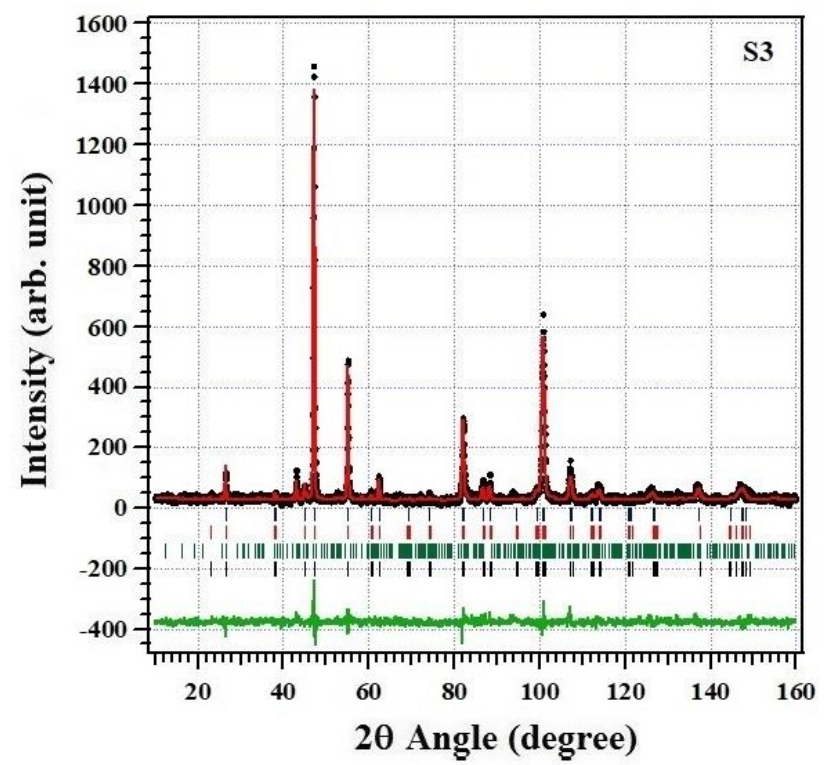

Figure 11. Neutron diffraction patterns of $\$ 3$, Rietveld analysis results based on the nuclear-and magnetic phase using basis functions of IRreps(3), with $\chi^{2}=1.7$.

Table 12. The coordinates of atomic fractions $\left(x_{j}, y_{j}, z_{j}\right)$, and atomic occupancy factor $\left(g_{j}\right)$ of $S 3$ sample based on neutron diffraction data.

\begin{tabular}{ccccc}
\hline Atom & $g_{j}$ & $x_{j}$ & $y_{j}$ & $z_{j}$ \\
\hline La & $0.6(1)$ & 0.0 & 0.0 & 0.25 \\
Ba & $0.23(1)$ & 0.0 & 0.0 & 0.25 \\
Mn & $0.6(2)$ & 0.0 & 0.0 & 0.0 \\
O & 1.0 & $0.4731(6)$ & 0.0 & 0.25 \\
\hline
\end{tabular}

Table 13. The coordinates of atomic fractions $\left(x_{j}, y_{j}, z_{j}\right)$, and atomic occupancy factor $\left(g_{j}\right)$ of $B_{a L a} \mathrm{O}_{4}$ phase based on neutron diffraction data.

\begin{tabular}{ccccc}
\hline Atom & $g_{j}$ & $x_{j}$ & $y_{j}$ & $z_{j}$ \\
\hline $\mathrm{Ba}$ & 0.164 & $0.220(3)$ & $0.660(2)$ & 0.25 \\
$\mathrm{La}(1)$ & 0.147 & $0.04(1)$ & $0.44(1)$ & 0.25 \\
$\mathrm{La}(2)$ & 0.367 & $0.651(7)$ & $0.584(6)$ & 0.25 \\
$\mathrm{O}(1)$ & 0.828 & $0.243(4)$ & $0.253(5)$ & 0.25 \\
$\mathrm{O}(2)$ & 0.636 & $0.414(7)$ & $0.032(5)$ & 0.25 \\
$\mathrm{O}(3)$ & 0.597 & $0.547(6)$ & $0.764(5)$ & 0.25 \\
$\mathrm{O}(4)$ & 0.593 & $0.022(6)$ & $0.123(4)$ & 0.25 \\
\hline
\end{tabular}




\section{CONCLUSIONS}

In this study, the $\mathrm{La}_{(1-x)} \mathrm{Ba}_{x} \mathrm{MnO}_{3}$ (LBMO) sample's magnetic ordering dependence on the Barium mole fraction has been verified by way of experimental investigation and analysis on its weak ferromagnetic property and its electromagnetic wave absorption characteristic. If the mole fraction of $\mathrm{Ba}$ in LBMO is smaller than 0.2 , the sample is classified as an antiferromagnetics, on the other hand if the mole fraction of $\mathrm{Ba}$ is greater than 0.2 the material is then classified as having a weak ferromagnetic property. Experimental evidence of the formation of weak ferromagnetic property of the LBMO in its unit cell could readily be obtained by using the neutron diffraction technique. However, in the frequency range between 8-12 GHz, the electromagnetic waves absorption of both antiferromagnetic and weak ferromagnetic compounds occurs at around $10.8 \mathrm{GHz}$ with the absorption of around $5 \mathrm{~dB}$. The mole fraction of Barium has also been found to affect the LBMO phase formation, the magnetic properties, and the electromagnetic waves absorption characteristics of LBMO.

\section{ACKNOWLEDGEMENT}

The authors thank the management of the Center for Science and Technology of Advanced Materials, which has provided the research facility. To colleagues in PSTBM especially Dr. Aziz Khan Jahya, thanks for their kind help.

\section{REFERENCES}

1. Naji, S., Benyoussef, A., El Kenz, A., Ez-Zahraouy, H., Loulidi, M. (2012). Monte Carlo study of phase transitions and magnetic properties of $\mathrm{LaMnO}_{3}$ : Heisenberg model. Physica, A391, 3885-3894.

2. Maria, B., Takaki, M., Mohammad, S., Ho-kwang, M., Lorenzo, M., Paolo, P., Sashi, S., and Viktor, V.S. (2015). Origin of colossal magnetoresistance in $\mathrm{LaMnO}_{3}$ manganite. Proc. National Acad Sci USA, 112 (35), 1086910872.

3. Chul-min, H., Min-sook, L., Seong-Cho, Y., Kyeongsup, K., Jaeyeong, K., and Bo Wha, L. (2010). Magnetocaloric effect of perovskite manganites of $\mathrm{La}_{0.8} \mathrm{~A}_{0.2} \mathrm{MnO}_{3}(\mathrm{~A}=\mathrm{Ca}, \mathrm{Sr}, \mathrm{Ba})$. Journal of the Korean Physical Society, 57(6), 1893-1896.

4. Ivan, M., Proloy, T.D., Max, D., James, G.S., Miguel, A.U-L., Saikat, D., Chennan, W., Matthias, R., and Christian, B. (2014). Influence of $\mathrm{La}$ and $\mathrm{Mn}$ vacancies on the electronic and magnetic properties of $\mathrm{LaMnO}_{3}$ thin films grown by pulsed laser deposition. Physical Review, B 89, 174422-1-174422-2.

5. Korotana, R., Mallia, G., Gercsi, Z., and Harrison, N.M. (2013). A hybrid-exchange density functional study of Ca-doped LaMnO 3. Journal of Applied Physics, 113, 17A910-1-17A910-3.

6. Yulan, C., Jianming, D., Xuebin, Z., Dajun, W., Yuping, S. (2010). Preparation, magnetic and microwave absorption properties of $\mathrm{La}_{0.5} \mathrm{Sr}_{0.5} \mathrm{MnO}_{3} / \mathrm{La}(\mathrm{OH})_{3}$ composites. Materials Research Bulletin, 45, 663-667.

7. Kalmykova, T.V., Tarapov, S.I., Neduch, S.V., Krivoruchko, V.N., Danilenko, I.A., Burchovetckii, V.V., Gurtovoj, G.G. Peculiarities of electromagnetic waves absorption in polymer magnetic nanocomposites ( $\mathrm{La}, \mathrm{Sr}_{\text {) }} \mathrm{MnO}_{3}$. (2012). Functional Materials, 19(4), 486-492.

8. Wael Saad Mohamed, A. (2014). Optical study of novel perovskitic oxides, with focus on their lattice and electronic properties. Ph.D. Thesis, Sapienza, University of Rome, p. 27.

9. Vasilii, Z. (2010). Magnetic and transport properties of $\mathrm{LaMnO}_{3+\delta}, \mathrm{La}_{1-x} \mathrm{Ca}_{x} \mathrm{MnO}_{3} \mathrm{La}_{1-x} \mathrm{Ca}_{x} \mathrm{Mn}_{1-y} \mathrm{Fe}_{y} \mathrm{O}_{3}$ and $\mathrm{La}_{1}$ ${ }_{x} \mathrm{Sr}_{x} \mathrm{Mn}_{1-y} \mathrm{Fe}_{y} \mathrm{O}_{3}$. Ph.D Thesis, Lappeenranta University of Technology, Lappeenranta, Finland, p. 17.

10. Alejandra Juliette Baena, V. (2014). The role of interfaces in heterostructures: Strained manganites and Silicon for quantum computing. Ph.D Thesis, Facultad De Ciencias Fisicas, Universidad Complutense De Madrid, Madrid, p. 34.

11. Priyo, S., and Wisnu, A.A. (2013). Structural, magnetic and electrical properties of $\mathrm{La}_{1-x} \mathrm{Ba}_{x} \mathrm{MnO}_{3}(x=0-0.7)$ for absorber electromagnetic wave. J. Basic. Appl. Sci. Res., 3(7), 230-234.

12. Sukirman, E., Wisnu, A.A., and Yustinus, P. (2012). Crystal structure and magnetoresistance of $\mathrm{La}_{0.7} \mathrm{Ca}_{0.3} \mathrm{MnO}_{3}$ perovskite at room temperature. Indonesian Journal of Nuclear Science and Technology, 13(2), 61-72.

13. Asma, K., Saadat, A.S., Affia, A. (2013). Synthesis and characterization of alkaline-earth metal (Ca, Sr, and Ba) doped nanodimensional $\mathrm{LaMnO}_{3}$ rare-earth manganites. Chin. Phys. Lett., 30(7), 077501-1- 077501-4.

14. Xianguo, L., Niandu, W., Pingping, Z., Nannan, B., Siu Wing, O., Caiyun, C., Yuping, S. (2015). Large scale synthesis of superparamagnetic face-centered cubic $\mathrm{Co} / \mathrm{C}$ nanocapsules by a facile hydrothermal method and their microwave absorbing properties. Materials Research, 18(4), 756-762.

15. Sukirman, E., Wisnu, A.A., Andon, I., Herry, M., Teguh Y.S. Panca, P., Aziz Khan, J., Dani, G. (2017). Neutron diffraction studies of the $\mathrm{La}_{1-x} \mathrm{Ba}_{\times} \mathrm{MnO}_{3}$ magnetic oxide. Presented in National Seminar on Nuclear Technology Empowerment, National Nuclear Energy Agency, November 21-22, p. 347, Puspiptek Serpong, Indonesia. 